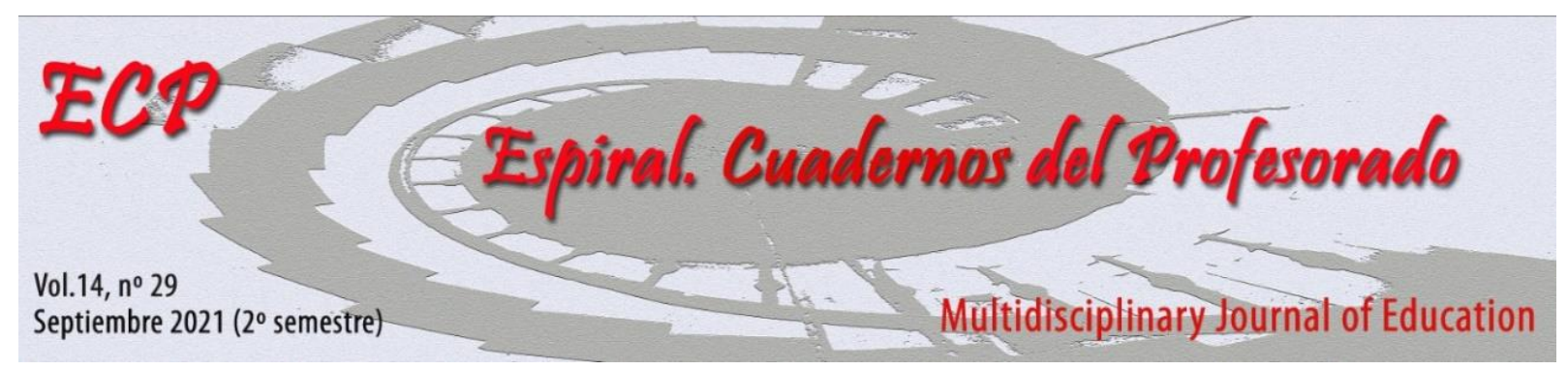

\title{
Enseñanza online: problemáticas en torno al aprendizaje, la educación lingüística y la desigualdad
}

\section{Online Teaching: Problematic around Learning, Linguistic Education and Inequality}

\author{
Nieves García Prados ${ }^{1}$ y Fernando Valverde Rodríguez ${ }^{1}$ \\ ${ }^{1}$ University of Virginia, Estados Unidos
}

\section{Resumen}

En los últimos años han proliferado las publicaciones y guías sobre las ventajas de la enseñanza online, sin que se haya hecho demasiada incidencia en las posibles problemáticas. Por eso, cuando un nuevo método educativo tiene que aplicarse en el aula a consecuencia de una pandemia mundial de coronavirus, se hacía necesario preguntarse cuáles han sido los retos y, especialmente las dificultades e inconvenientes que han experimentado los profesores desde su punto de vista y experiencia. A través de una encuesta a docentes de más de una veintena de universidades e instituciones educativas públicas y privadas, tanto de los Estados Unidos como de Europa y Latinoamérica, hemos recopilado sus opiniones y comentarios en torno a los desafíos que plantea este tipo de enseñanza, centrándonos en la capacidad de aprendizaje, las competencias educativas, y, especialmente, en las desigualdades detectadas entre el alumnado.

Palabras clave: Educación Lingüística; Lengua y Literatura; Enseñanza online; Didáctica.

\begin{abstract}
In recent years, publications and guides on the advantages of online teaching have proliferated, without much emphasis on the possible problems. For this reason, when a new type of educational method has to be implemented in the classroom as a result of the global coronavirus pandemic, it has become necessary to ask ourselves about the challenges and, especially, the difficulties and inconveniences that teachers have confronted from their own experience. Through a survey sent to professors of more than twenty universities both public and private in the United States and in Europe and Latin America, we have compiled a list of their opinions and comments regarding the challenges posed by this type of teaching, focusing on the learning capacity, educational competencies, and, especially, on the inequalities detected among students.
\end{abstract}

Keywords: Linguistic Education; Linguistics and Literature; Online Teaching; Didactics.

Fecha de recepción: 14/07/2021

Fecha de aceptación: 04/08/2021

Correspondencia: Fernando Valverde Rodríguez, University of Virginia, Estados Unidos Email: fv7vh@virginia.edu 


\section{Introducción}

En una entrevista publicada en 2021 por la Revista Poéticas, el hispanista Allen Josephs declaraba que en su informe anual sobre enseñanza para su universidad, la West Florida University, se preguntaba si sería posible imaginar a Sócrates, Aristóteles, Buda o Jesucristo enseñando online. Lejos de querer compararse con ellos, Josephs ponía sobre la mesa una de las problemáticas más actuales en la educación. "Pienso que los mejores modelos para la enseñanza pertenecen a la enseñanza peripatética, en grupos pequeños. Hemos sustituido a los maestros por la tecnología y el resultado es cada vez peor. Los estudiantes, cada año, tienen peores resultados, menos ganas de trabajar, menos conocimiento y menos lectura", sentenciaba Josephs (2021, p. 117), quien este mismo año recibió una condecoración por sus 50 años en el aula de enseñanza universitaria y fue nombrado presidente honorífico de SAMLA, una de las mayores asociaciones de profesores de lenguas modernas en los Estados Unidos.

Son muchos quienes como Josephs se resisten a ver en la enseñanza online una opción frente a la educación presencial. Algunos incluso han renunciado a sus puestos de trabajo, desencantados por el nuevo modelo. Otros se han visto empujados a aprender rápidamente lo que se suponía una solución de emergencia a causa de la pandemia provocada por el coronavirus. También hay quien se muestra entusiasta con el nuevo modelo por diferentes razones. En este artículo hemos querido recoger la opinión de diferentes profesores de distintas universidades públicas y privadas de Estados Unidos, Latinoamérica y de Europa para detectar algunas de las dificultades que plantea la enseñanza online, sus retos y sus desafíos.

Cuando Josephs se refiere a la enseñanza peripatética nos indica una dirección concreta: la antigua

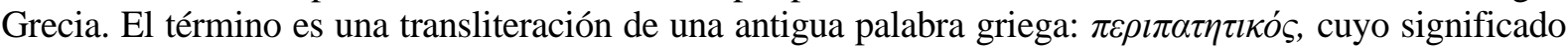
era "caminar" o "aquellos que caminaban". Durante siglos fue empleada para designar a los seguidores de Aristóteles, discípulo a su vez de Platón y Sócrates. Josephs se refiere a una enseñanza en la que la experiencia real ocupe un lugar protagonista, tanto para el conocimiento filosófico como para el científico.

Antes de la pandemia del coronavirus, la mayor parte de nuestras clases ya se habían convertido en "Smart Classrooms" o lo que académicos como Baepler y Walker (2014) han denominado "Active Learning Classrooms", lo cual había reconfigurado su espacio y su experiencia tanto para los docentes como para los estudiantes. Estas clases híbridas, en las que el profesor interactúa con la tecnología, convirtió a esta última en un instrumento. Sin embargo, el inicio de la pandemia en marzo de 2020 obligó a la enseñanza a distancia, convirtiendo a la tecnología no ya en un instrumento, sino en el intermediario.

Tras tres décadas de experiencia en la educación superior, Claire Howell Major, profesora en la Universidad de Alabama, escribió un libro titulado Teaching online, de gran difusión en los Estados Unidos. Major considera que cuando un profesor está haciendo su trabajo online la tecnología no sólo sirve como un instrumento funcional que puede ayudar al aprendizaje, sino que se sirve como intermediario entre nuestras realidades, al mismo tiempo que pasa a formar parte de ellas: "Al enseñar en línea, nuestras realidades educativas tienen como intermediaria a la tecnología. Es decir, la tecnología se erige como una interfaz entre nosotros y nuestro ámbito educativo; La percepción que tenemos de la realidad se ve alterada por esa tecnología" (Major, 2015, p. 9). La pregunta que nos planteamos, por tanto, es de qué manera ha influido ese intermediario en la enseñanza superior y cuáles han sido sus mayores inconvenientes desde el punto de vista de los docentes.

\section{Experiencias en los EEUU, Europa y Latinoamérica}

\section{Encuestas a Docentes}

Para nuestro trabajo, hemos realizado encuestas a más de una veintena de profesores de lengua y literatura de universidades de los EEUU (de estados como California, Kentucky, Virginia, Florida, Georgia, y Washington, entre otros); Europa (España, Reino Unido e Italia); y Latinoamérica (Bolivia, México y Colombia), quienes destacaron, en primer lugar, que el papel de intermediario que desempeña la tecnología entre el profesor y sus estudiantes se ha revelado "fallido". Palabras como "frialdad", "distancia", "sensación de vacío" o "incomunicación" han servido para definir, a través de las respuestas ofrecidas por los profesores, la tecnología que ha hecho posible continuar con las "clases" cuando reunirse suponía un riesgo para la salud.

Espiral. Cuadernos del Profesorado | ISSN 1988-7701 | 2021, 14(29), 56-64 
Para Jeremy Paden, profesor de la Universidad de Transylvania en Kentucky, una de las principales consecuencias de la enseñanza vía Zoom o cualquier otra plataforma similar es la disminución del compañerismo. La conversión del espacio del aula en un espacio virtual modifica los roles de quienes ocupaban dicho espacio. Ahora los estudiantes, al no poder conocerse entre sí, apenas interactúan entre ellos. La sensación de pertenencia al grupo desaparece y es la individualidad la que queda expuesta, por un lado la del docente y por el otro la del estudiante. Paden cita a Marshall McLuhan para explicar cómo la telecomunicación "es un medio frío que requiere un mayor esfuerzo por parte del estudiante para procesar la información". Paden considera que se hace mucho más difícil interactuar en un aula virtual que en una real y coincide, por tanto, con la definición realizada por Krashen (1983) sobre "el filtro afectivo" o cómo la actitud del que aprende, y sus sentimientos, su estado anímico y otro tipo de elementos emotivos, influyen en la adquisición de aptitudes dentro del aula.

En el aula virtual, los estudiantes van a demandar continuamente la presencia del profesor, en el sentido de que éste se muestre activo todo el tiempo, siendo el factor que tendrán más en cuenta a la hora de valorar el trabajo docente, su grado de satisfacción. "Dado que los instructores y los estudiantes son seres sociales, el aula en línea debe adaptarse a través de un lenguaje que aborde la presencia social, la presencia del instructor y la presencia cognitiva (Lehman \& Conceição, 2010, 315-317). Es decir, una consideración pedagógica importante para fomentar la participación de los estudiantes en línea es mediante la creación de un sentido de "presencia" en el que los estudiantes tengan la sensación de "estar allí" y "estar juntos" en el entorno en línea. Pero, pese a que exigen más, arriesgan menos. Paden explica cómo en sus clases la participación ha disminuido de forma radical. En el mismo sentido el profesor Gordon E. McNeer, de la Universidad de North Georgia, considera que las diferentes normas impuestas por el nuevo sistema online han tenido como consecuencia la disminución de la participación. Una y otra vez se repite la imagen de las pantallas en negro con el micrófono apagado, lo cual condena al profesor a una suerte de soledad frente a la cámara.

Aunque pueda resultar paradójico, en el mundo de la conexión online precisamente lo que falla es eso. "Las interacciones personales sufren en el medio electrónico. Hay poca risa, y poca conexión”, explica Ricardo Padrón, profesor de la Universidad de Virginia.

"Los estudiantes se escudan en el anonimato que les permiten algunas plataformas", asegura Aurora Martínez Ezquerro, de la Universidad de La Rioja, para quien la disminución de la participación ha supuesto un deterioro de la enseñanza pues "la presencialidad del profesor no se suple de forma completa" y se reduce la "función mediadora" del docente (Lanseros y Sánchez, 2017). En esta misma dirección inciden tanto Francisco José Sánchez, profesor de la Universidad de Granada, para quien "en general ha disminuido la participación" como Ricardo Padrón, profesor de la Universidad de Virginia, quien ha podido comprobar cómo "asisten, pero no siempre participan, por lo menos no tanto como en las clases presenciales".

Isabel Balseiro, del Harvey Mudd College de California, va más allá al considerar que la participación no disminuye de una forma horizontal, sino vertical: son los más tímidos, los que tienen más problemas para comunicarse, los que menos participan. Esta circunstancia provocaría precisamente una situación de vulnerabilidad en el nuevo espacio virtual del aula en el que algunos estudiantes quedarían marginados o incluso aislados sin que por el momento los profesores tengan los recursos o las herramientas necesarias para evitarlo.

\section{El Resultado: Desigualdad Digital, Discriminación Real}

Esta dificultad para comunicarse sería sólo la primera de las desigualdades descritas por los profesores entrevistados, pues la inmensa mayoría de ellos consideran de una forma u otra que la enseñanza online amplifica las desigualdades entre los estudiantes. Anthony L. Geist, profesor de la Universidad de Washington con más de cuarenta años de experiencia, considera que el nuevo modelo tecnológico discrimina de diferentes formas. "Algunos estudiantes viven con su familia en casas pequeñas sin acceso a un espacio privado para conectarse. Aunque todos mis alumnos tienen acceso a internet, algunos no siempre tienen buena conexión. Otros se han visto obligados a conectarse desde el coche rumbo a su trabajo 
o en otras situaciones que no son ideales", explica Geist, para quien hay un elemento de clase social que hace sacar ventaja a unos alumnos sobre otros.

La profesora Karen Stolley, de la Universidad de Emory, en Atlanta, Georgia, identifica múltiples desigualdades que se han visto ampliadas por la enseñanza online, refiriéndose principalmente a "las sociales, las psíquicas y las materiales". La profesora Rosi Song, de la Universidad de Durham en el Reino Unido, coincide con este análisis en el sentido de que "la parte física que se necesita para participar de manera productiva en un formato online ha perjudicado a muchos estudiantes". Para la profesora Remedios Sánchez, de la Universidad de Granada, este hecho es algo inevitable en cuanto a la implicación material que la tecnología arrastra. "Los alumnos dejan de tener un acceso universal, que garantice el desarrollo igualitario del curso", lo cual tiene que ver con una cuestión de clase social que ha sido apenas entrevista en los Estados Unidos, intuida en Europa y convertida en evidencia en países con menos recursos económicos.

El profesor Gabriel Chávez imparte clases de literatura en la Universidad Privada de Santa Cruz, en Santa Cruz de la Sierra, la mayor ciudad de Bolivia, con más de dos millones de habitantes. Para Chávez, no existe duda alguna de que la enseñanza online ha supuesto una brecha de posibilidades entre los que sí y los que no, entre los que tienen y los que no tienen. "Los estudiantes de universidades y colegios privados tienen muchas más posibilidades de disfrutar de una conexión permanente a Internet con una buena velocidad. En cambio, ha sido muy difícil que los estudiantes de educación pública puedan seguir estudiando por la pandemia. Cuanto más pobres sean y más alejados vivan de las ciudades, los estudiantes tendrán menos posibilidades de acceder a la educación virtual", explica Chávez, a quien no sorprende que su gobierno tomara la decisión en 2020 de clausurar el curso escolar a mitad de año con promoción automática de curso para todos los estudiantes, porque la educación pública no podía continuar de manera virtual $^{1}$. "Los colegios públicos siguieron trabajando con acuerdos extralegales con padres de familia, pero incluso muchos padres de clase media tuvieron que dejar de educar a sus hijos en colegios o universidades privadas porque la crisis económica que causó la pandemia les hizo perder sus trabajos o su capacidad de pago", añade Chávez, que vivió la experiencia de que algunos de sus estudiantes tuvieran que abandonar la clase. "Seguramente pertenecían a familias afectadas por la crisis económica. No hay duda de que la enseñanza virtual derivada de la pandemia ha ahondado la brecha que separa a unas instituciones educativas de otras, a unos estudiantes de otros", concluye.

Nuestra perspectiva, que tomó como ámbito preferente los Estados Unidos y Europa, se diluye por sobre un pedestal de privilegios apenas miramos hacia otros países latinoamericanos. Basta con atravesar la frontera entre Estados Unidos y México, con sus 3169 kilómetros, 1954 millas. Alí Calderón, profesor de la Benemérita Universidad Autónoma de Puebla, asegura que para él ha sido imposible "sostener el diálogo" ya que existe una merma en el compromiso que sí está presente en la enseñanza presencial, el famoso engagement ha saltado por los aires en muchos casos concretos de estudiantes que estaban conectados en la enseñanza presencial y que han pasado a la desidia. Calderón considera que en parte se debe a lo que Foucault llamaba "violencia simbólica" cuando se refería a un sacerdote o a un maestro que ostenta una posición de poder o autoridad que puede ejercerse de manera positiva desde el carisma o el refuerzo de las emociones y los estímulos. Sin embargo, lo que Calderón ha detectado en sus cursos es bien diferente. "La mayoría de los alumnos apaga sus cámaras. Otros no se conectan. ¿Por qué? En el caso de México puede suponerse que viven en sitios sin cobertura de Internet, que no cuentan con una computadora para seguir la clase o que el espacio no es el adecuado (posiblemente debido al hacinamiento) o a dificultades familiares. También existe la posibilidad de la simple apatía porque se relaja la autoridad simbólica que ejerce con su mero estar el profesor en el modo presencial", argumenta Calderón, para quien la enseñanza online genera un espacio de dispersión que es lo opuesto al significado simbólico del aula.

\footnotetext{
1 "La gran mayoría del área rural no cuenta con internet, los niños no tienen internet. La fibra óptica sólo llega a las ciudades. No hay condiciones... por lo que hemos visto conveniente clausurar el año escolar”, declaró el ministro de la presidencia, Yerko Núñez, el 2 de agosto de 2020, como recogieron varios medios latinoamericanos, como La Nación de Argentina o La Jornada, en México.
} 
Similar al contexto mexicano es el de Colombia. Federico Díaz-Granados es profesor en una de las instituciones educativas de élite de Bogotá, el Gimnasio Moderno. Pese a que la mayor parte de sus estudiantes provienen de familias con recursos, Díaz-Granados no tiene ninguna duda de que la enseñanza online ha extremado las desigualdades. "En un país como Colombia, donde hay un grueso de la población sin capacidad de tener un ordenador, que vive en lugares y regiones sin cobertura de WiFi, se han evidenciado mucho más las brechas sociales. ¿Qué ocurre con los hogares donde solo hay un ordenador y cinco hijos en edad escolar?", como respuesta están, por desgracia, las miles de historias de fracaso o abandono escolar.

Sobre su experiencia como profesor online, Díaz-Granados no duda en asegurar que ha sido negativa. "La educación tiene una base muy grande en las emociones y las sensaciones de lo que ocurre en una clase presencial"; por una parte, estas emociones sirven de estímulo y de refuerzo para conseguir que los jóvenes permanezcan atentos, mientras que la clase a través de plataformas informáticas ha supuesto "una dispersión" de los estudiantes que realizan otras actividades ocultándose tras "la cámara pagada", explica el docente colombiano. "Eso ha sido refugio de los tímidos, pero también de los estudiantes complicados. Hay que tener en cuenta que el umbral de atención de un estudiante online no supera los 7 minutos. Entonces el profesor debe convertirse en un animador que debe ingeniarse entretenidas actividades para conservar el nivel de atención un poco más tiempo", concluye no sin antes señalar la mayor de las frustraciones: aunque el esfuerzo es más grande, el rendimiento es menor. "La enseñanza online ha aumentado el cansancio de los estudiantes y de los profesores. Todos los colegas profesores de colegios y universidades han coincidido desde el comienzo de la pandemia que terminan la jornada mucho más agotados y con la sensación de que los estudiantes aprendieron menos", concluye.

\section{Evaluaciones y Posibles Plagios}

Pero de regreso al contexto de los Estados Unidos y Europa, la mayor parte de los profesores son conscientes de que algunos estudiantes están en una peor posición que otros aunque en muchos casos estas desigualdades sean difícilmente identificables. Frente a este hecho, lo primero que cabe preguntarse es cómo puede el docente valorar esta circunstancia en algo tan elemental como es una calificación justa al final del semestre que no cree una nueva desigualdad en los expedientes académicos.

Aurora Hermida-Ruiz, profesora del español en la Universidad de Richmond, asegura no haber podido establecer los mismos parámetros de calificación que acostumbraba en las clases presenciales, entre otros motivos por una mayor dificultad para detectar los plagios. Sorprendentemente no son pocos los docentes que se han visto desbordados por este aspecto. También la profesora Song apunta en esta dirección. "Ahora que todo se hace virtualmente y los estudiantes están manejando materiales online, estoy descubriendo más casos de usos indiscretos". La doctora Remedios Sánchez también ha notado que algunos estudiantes pueden tratar de sacar ventaja de ello sin que sea posible demostrarlo. "Estudiantes que habitualmente no asisten a clase han podido dejar el ordenador conectado aunque no estén asistiendo realmente, dado que no podemos obligarlos a encender la cámara", concluye.

También Jeremy Paden está convencido de que "el estudiante que quiera hacer trampa puede hacerlo con mayor facilidad". Todas estas circunstancias hacen muy complicada la calificación de una forma justa y segura. Por lo general, los profesores entrevistados han tenido que recurrir a sistemas de calificación diferentes de los que estaban acostumbrados a emplear cuando las clases eran presenciales. "Les exijo menos, en reconocimiento de las circunstancias extraordinarias", señala Geist, que coincide con McNeer en que hay una "mayor flexibilidad" a la hora de calificar, precisamente en un intento por parte de los profesores de "corregir las deficiencias del sistema".

En la búsqueda de una solución que sirva para remediar la frialdad tecnológica del intermediario, la profesora Karen Stolley, de la Universidad de Emory, considera que ha sido menos rigurosa con sus estudiantes que en otros cursos. "Debido a las circunstancias, me ha parecido lo más ético y lo más humano". Stolley, una de las profesoras más queridas y valoradas de su universidad, que incluso convoca un premio con su nombre para reconocer la divulgación del español, con toda sinceridad reconoce haber cambiado el formato de los exámenes y haber sido más flexible en diferentes aspectos, como las fechas límite de entrega de trabajos o actividades. "También he sido más generosa con las calificaciones... 
siempre pensando en la mejor manera de apoyar la educación de mis estudiantes a largo plazo sin quedarme atada a criterios estrechos de materia y expectativas", concluye.

No sólo Stolley ha variado su habitual método de calificación en busca de uno que pudiera reflejar de una forma más clara las desigualdades creadas. Otro ejemplo es el de Isabel Balseiro, que dice haber aumentado el número de actividades extracurriculares para aquellos estudiantes que quisieran hacerlas voluntariamente. "Este mecanismo hizo que quienes eran menos dados a participar durante la sesión de Zoom pudieran expresarse sin la presión del tiempo y de la cámara. Aunque me ha dado más trabajo, creo que es adecuado poder ofrecer las mismas oportunidades a un alumno con marcadas diferencias socioeconómicas, étnicas o de personalidad”, explica, contradiciendo una de las teorías expuestas por Stephen D. Brookfield en su defensa de la enseñanza online: que los estudiantes que tienen dificultades de expresión, aquellos más introvertidos, o que necesitan más tiempo para procesar la información y crear significados, se sienten menos intimidados por una cámara que por el escenario de una clase en vivo (Brookfield, 2015) ${ }^{2}$.

Aunque las guías de enseñanza online que han proliferado como un virus señalen el espacio virtual como garante de la igualdad, el mismo Allen Josephs no tiene duda alguna sobre la validez de la práctica propuesta por Balseiro e incluso se atreve a señalar un fenómeno como consecuencia de las contracciones del sistema, lo que podría denominarse la inflación gradual. "El sistema genera desigualdad, provoca ansiedad, disminuye la concentración de los estudiantes y de los profesores. Se ofrece un producto de enseñanza en el sentido más pobre del término, por lo que el nivel de exigencia no puede ser el mismo. La única opción que tienen los profesores es ser condescendientes con las evaluaciones u ofrecer múltiples oportunidades. Todo ello provoca que sea casi imposible que un alumno no obtenga una A final, lo que acabará por devaluar las calificaciones".

\section{Zoom Fatigue y Otros Síndromes}

Además de las desigualdades impuestas por el déficit de recursos, ¿qué otros factores pueden afectar a las clases a través de una pantalla? Desgraciadamente, nuestros estudiantes pertenecen a una generación que ya pasaba demasiado tiempo mirando un Smartphone, ya fuera en el autobús, en una cafetería o incluso mientras caminaban. Las clases propiciaban una desconexión, un vínculo con la realidad. Fernando Operé, profesor de la Universidad de Virginia, que en general considera muy útil la enseñanza online y encuentra en ella importantes beneficios, sí que ha detectado un cansancio en sus estudiantes. "Es posible que este cansancio provenga de que ha disminuido su vida social, y por tanto el estado de ánimo general", argumenta Operé. Habría por tanto un elemento psicológico que tiene que ver con estar encerrados, con la imposibilidad de abandonar un lugar, con la creación de un espacio artificial. Desgraciadamente, el contexto de la pandemia mundial nos ha dado muchas muestras de ello.

El propio Centers For Disease Control and Prevention (CDC) de los EEUU publicó una serie de advertencias sobre la salud con relación a las consecuencias que el confinamiento y la falta de contacto social (la conocida como distancia social) puede afectar a algunas personas. Entre los efectos más comunes, se refiere al miedo y a la preocupación por la salud propia y la de los otros, lo que unido a la posible pérdida de ingresos conlleva un estrés que puede derivar en diferentes patologías. Además, el CDC considera que este aislamiento provoca cambios en el sueño, en la alimentación, dificultad de concentración y en la toma de decisiones, pesadillas y problemas de sueño, empeoramiento de problemas de salud crónicos o de las condiciones de salud mental, un incremento en el uso de sustancias como el alcohol o el tabaco y reacciones físicas como dolores de cabeza, corporales, problemas de estómago y erupciones cutáneas ${ }^{3}$.

\footnotetext{
${ }^{2}$ Concretamente, Brookfield señala: "Los estudiantes que tienen problemas con el lenguaje, que son introvertidos o que necesitan tiempo para procesar información y crear sentido, así como aquellos que se sienten intimidados por el contexto del aula (en particular, tener que desempeñar el papel de estudiante inteligente, capaz y comprometido) se benefician de la privacidad del aprendizaje en línea” (2015, p. 171).

${ }^{3}$ En su página web www.CDC.gov encontramos recomendaciones sobre cómo lidiar de "manera saludable" con el estrés provocado por la pandemia de coronavirus: "La pandemia de COVID-19 ha tenido un efecto importante
} 
La ansiedad, que ocupaba un lugar cada vez más central en la vida de los ciudadanos modernos, se ha multiplicado al mismo ritmo que la soledad. "El cansancio de los estudiantes es mucho mayor. Al estar frente a un medio de comunicación frío, se ven obligados a prestar más atención a la vez que no reciben el sentimiento de bienestar que supone estar junto a otras personas. De hecho, la enseñanza online puede agravar los sentimientos de aislamiento", explica Jeremy Paden, en consonancia con lo expuesto por Nauzet Lozano, profesor de secundaria en las Islas Canarias, para quien el cansancio no ha tenido que ver únicamente con la relación con un mundo frío, sino especialmente con la imposibilidad de crear espacios seguros de desconexión. "La enseñanza en línea provoca la sensación de estar conectado constantemente, en cualquier momento puede llegar un mensaje o una tarea, un correo electrónico, un material inesperado... Es posible que nunca podamos desconectar del todo", asegura.

También Aurora Hermida, Rosi Song, Isabel Balseiro o Anthony Geist coinciden en que el incremento del cansancio en los estudiantes es uno de los factores más negativos: la mayor parte de los estudiantes parecían más cansados de lo normal, en algunos de los casos incluso daban la impresión de estar agotados. Geist alude al denominado como Zoom fatigue, que ha sido definido por la Dra. Jena Lee, profesora de la escuela de Medicina de la Universidad de Los Ángeles, como una afectación psicológica que provoca cansancio, preocupación y agotamiento asociados con el uso excesivo de las plataformas virtuales de comunicación. Entre las múltiples causas de esta fatiga, Lee (2020) alude al hecho de que inevitablemente existe un retraso en el sonido que es percibido por el cerebro, aunque sea de microsegundos, lo que afecta a nuestras respuestas verbales visuales de forma negativa, incluso sin problemas técnicos o de internet. También la Dra. Brenda Wiederhold, presidenta del Virtual Reality Medical Center en los Estados Unidos, defiende que el llamado "aprendizaje sincrónico" en realidad no es "sincrónico". "Nuestros cerebros están acostumbrados a captar el lenguaje corporal y otras señales, eso sin mencionar los aumentos de dopamina que se experimentan durante la comunicación cara a cara. En una videollamada, algo está fallando y nuestro subconsciente está reaccionando a ello. La comunicación no es en tiempo real, aunque podamos creer que sí” $(2020)^{4}$.

Por si fuera poco, muchos de los estudiantes son nativos digitales que apenas diferencian la experiencia de una clase con el uso de una red social, lo cual vacía de contenido parte del discurso. "Hablar a través de las redes sociales en realidad no tiene nada que ver con hablar (...) Cada vez entendemos menos lo que les pasa a los otros. Se está destruyendo nuestra capacidad de empatizar (...) Recuerdo cuando se suponía que Internet traería consigo una sociedad transparente. Ha ocurrido todo lo contrario", explica Jaron Lainer, uno de los gurús de las nuevas comunicaciones de Silicon Valley (2018, p. 100).

\section{Afectación de Competencias Lingüísticas}

Por último, para concluir este breve recorrido por los problemas de la enseñanza online, quisimos preguntar a profesores que enseñan una segunda lengua si alguna de las competencias lingüísticas se había visto afectada de forma especial, siguiendo la división estándar de las mismas: competencia lectora, competencia del habla, competencia de la comprensión y competencia de escritura.

"No hay una sola competencia lingüística que no se haya visto afectada de forma negativa. Es una realidad muy preocupante", señala Gordon E. McNeer, doctor por Princeton y uno de los profesores más experimentados de los Estados Unidos en la enseñanza del español como segunda lengua.

Para María Felisa Bermejo, de la Universidad de Turín, la producción oral es la competencia que se ha visto más afectada, especialmente si un grupo era demasiado grande. En el mismo sentido, Jeremy Paden considera que "un aula típica de adquisición de lengua debería ser un lugar de mucha producción...

en nuestras vidas. Muchos de nosotros enfrentamos desafíos que pueden ser estresantes, abrumadores y causar emociones fuertes en adultos y niños. Las acciones de salud pública, como el distanciamiento social, son necesarias para reducir la propagación del COVID-19, pero pueden hacernos sentir aislados y solos y pueden aumentar el estrés y la ansiedad".

${ }^{4}$ Declaraciones recogidas en un artículo publicado por la National Education Association de los EEUU, con el título "How 'Zoom Fatigue' Impacts Communication With Students".

Espiral. Cuadernos del Profesorado | ISSN 1988-7701 | 2021, 14(29), 56-64 
pero la comunicación por internet no permite que muchos hablen a la vez". También Fernando Operé cree que los estudiantes estaban "más cohibidos al hablar".

Sobre la escritura, Karen Stolley ha necesitado de un trabajo "extra" para suplir las dificultades que la enseñanza online supone para la adquisición de esta competencia, que tiene mucho que ver con el diálogo y con la elaboración de un borrador, cuya corrección y discusión en el mundo virtual son más lentos.

En lo que se refiere a la competencia de la comprensión, Gustavo Pellón, de la Universidad de Virginia, detecta una ventaja en la enseñanza online. "La experiencia es mejor que en clase. Me oyen mejor y yo los oigo mejor en la mayoría de los casos". También Aurora Hermida cree que entienden mejor porque existe "una mayor facilidad para leer los labios".

Con respecto a la competencia lectora, el propio Josephs fue muy crítico en la entrevista que ha dado pie a este recorrido. "Leen menos que nunca", a lo que habría que añadir que "leen peor", precisamente por el trabajo que supone leer todo el tiempo en una pantalla. "Ese desapego a los libros es un tema del que tendremos que hablar más adelante, pero que cada día aleja más a nuestros estudiantes no sólo de la literatura, sino de cualquier forma de cultura o aprendizaje. Al estar todo disponible online, el conocimiento también se devalúa y las implicaciones de esto aún no las conocemos, pero no soy nada optimista", sentencia Josephs.

\section{Conclusiones}

Lejos de pretender ofrecer un panorama catastrofista, en este artículo hemos tratado de recoger algunos de los inconvenientes con la esperanza de que futuros estudios puedan analizarlos con mayor profundidad para proponer rectificaciones que ayuden a hacer más productivas las herramientas que la tecnología pone al alcance de los docentes. Como señala el profesor Federico Díaz-Granados, en general sentimos que el mundo no estaba preparado para una educación $100 \%$ online. Fue una solución de emergencia que nos permitió continuar trabajando en un tiempo de condiciones excepcionales, de miedo, enfermedad y muerte, de incertidumbre y soledad. Fue una herramienta que podría haber fomentado la autonomía y la confianza en el trabajo, pero en muchos casos los procesos individuales de aprendizaje han terminado siendo el espacio ideal para la ambigüedad. "La enseñanza online ha sido el recurso de un tiempo mediocre que se ha visto sorprendido por una pandemia y que en medio de muchas improvisaciones y sin estar preparados para una verdadera educación virtual se refugió en una forma de acomodarse a un tiempo de indecisiones y de azares", explica Díaz Granados.

Somos conscientes de que igual que existen argumentos en contra de la enseñanza virtual también existen argumentos a favor o incluso entusiastas de ella. Una visión muy optimista ha sido aportada por otros colegas, que consideran que la enseñanza online puede ser incluso superior a la presencial. Con respecto a la participación de los estudiantes, el profesor Gustavo Pellón aseguró que el uso de breakoutrooms "ha fomentado la participación de los estudiantes y el intercambio animado entre ellos", hasta el punto de que calificar el resultado de "conmovedor".

La profesora Raquel Lanseros, de la Universidad de Zaragoza, no sólo no tiene la sensación de que el grado de aprendizaje haya sido menor, sino que considera que la participación y la asistencia han aumentado con respecto a las clases presenciales y que las razones de la desigualdad no hay que buscarlas en el soporte, sino "en decisiones económicas y políticas muy concretas, preponderantes en las últimas décadas en el mundo occidental".

Sea o no esto cierto, en los últimos años han proliferado los "especialistas" y "gurús" dedicados a escribir guías sobre las ventajas de este nuevo tipo de enseñanza (una variante de la enseñanza a distancia que antes se había proyectado por otros medios como la TV o la radio y cuyo éxito nunca logró ser demostrado). En ellas se nos ofrecen consejos sobre cómo convertirnos en profesores virtuales, algunos de ellos útiles, otros no tanto.

En este artículo hemos tratado de reflejar la problemática de desigualdad, atención, participación, evaluación y compromiso desde muy diferentes perspectivas, sin enumerar siquiera los beneficios que podrían derivarse de una enseñanza o de otra. 
Para lo contrario sería recomendable consultar una de las guías más populares sobre la enseñanza online, escrita por Susan Ko, de la Universidad de Maryland, y por Steve Rossen, de la Universidad de California, en la que se asegura sin complejos de ninguna clase que lo que convierte en única a la enseñanza online es que no hay que estar en un lugar concreto para enseñar. "No tienes que llevar un maletín lleno de papeles o el ordenador portátil a una clase, detenerte en un atril, garabatear en la pizarra o calificar en un aula congestionada mientras tus estudiantes toman un examen. Ni siquiera hay que sentarse en la oficina esperando a que los estudiantes se presenten para una reunión" (Ko y Rossen, 2017, p. 4).

Robert Frost, de entre los dos caminos que divergieron en su bosque amarillo, eligió el menos transitado y eso fue lo que marcó la diferencia. En Andalucía, un refrán dice que el camino más largo y difícil es el que conduce al éxito. Sean aplicables o no estos consejos a la didáctica, lo que consideramos esencial es poner el foco en los estudiantes y en la importancia de la enseñanza, no en una mayor comodidad del profesorado.

Contribución de cada Autor: Conceptualización, García Prados y Valverde Rodríguez; metodología, García Prados y Valverde Rodríguez; análisis, García Prados y Valverde Rodríguez; escritura del manuscrito, Valverde Rodríguez; escritura, revisión y edición, García Prados.

Financiación: Esta investigación no recibió ninguna financiación externa.

Agradecimientos: A todos los docentes participantes que accedieron a contestar la encuesta para este estudio.

Conflicto de Intereses: Los autores declaran que no tienen conflicto de intereses.

\section{Referencias}

Brookfield, S. D (2015). The Skillful Teacher: On Technique, Trust, and Responsiveness in the Classroom, John Wiley \& Sons, Incorporated.

Baepler, P. \& Walker, J.D. (2014). Active Learning Classrooms and Educational Alliances: Changing Relationships to Improve Learning. New Directions for Teaching and Learning.

Centers For Disease Control and Prevention (CDC) (1 de diciembre de 2020) Coping with Stress. (https://www.cdc.gov/coronavirus/2019-ncov/daily-life-coping/managing-stress-anxiety.html).

Josephs, A. (2021). En García Prados, Nieves. "Entrevista a Allen Josephs", Revista Poéticas, 12, p. 111-117. Recuperado de http://poeticas.org/index.php/poeticas/article/view/204.

Ko, S. \& Rossen, S. (2017). Teaching online: A Practical Guide. Routledge Press.

Krashen, S. D. \& Terrel, T. D. (1983). The Natural Approach: Language Acquisition in the Classroom. Pergamon.

Lanier, J. (2018). Diez Razones para borrar tus redes sociales de inmediato. Debate.

Lanseros, R. \& Sánchez García, R. (2017). "La poesía de Joan Margarit para nivel avanzado de EEOOII en E/LE: una propuesta didáctica". Espiral, Cuadernos del Profesorado. 10(21), 90-99. https://doi.org/10.25115/ecp.v10i21.1032

Lee, J. A (17 de noviembre de 2020) "Neuropsychological Exploration of Zoom Fatigue". Psyquiatric Times. Recuperado de https://www.psychiatrictimes.com/view/psychological-exploration-zoom-fatigue.

Lehman, R. M., \& Conceição, S. C. O. (2010). Creating a Sense of Presence in Online Teaching: How to "be There" for Distance Learners. Jossey-Bass

Major, C. H (2015). Teaching Online: A Guide to Theory, Research, and Practice. Johns Hopkins University Press.

National Education Association (16 de octubre de 2020). How 'Zoom Fatigue' Impacts Communication with Students https://www.nea.org/advocating-for-change/new-from-nea/how-zoom-fatigue-impacts-communicationstudents. 